\title{
OUTCOME OF PLATE OSTEOSYNTHESIS IN THE MANAGEMENT OF PROXIMAL HUMERAL FRACTURES IN ADULTS
}

J. M. Muthuuri, FCS (ECSA), MBChB, MMed (Surg.), H. Dip. Orth (SA), Consultant Orthopaedic and Trauma Surgeon, The Mombasa Hospital Private Clinics, P.O. Box 84074, Mombasa, Kenya.E-mail: michenimuthuuri@yahoo.com

\section{ABSTRACT}

Background: This paper reports the aetiology, presentation and management of proximal humeral fractures (PHF) in our setup.

Objective: To share our experiences with this relatively uncommon injury. The secondary objective is : to assess the functional outcome of plate osteosynthesis in management of displaced proximal humerus fractures (displacement of $>5 \mathrm{~mm}$ or/ and angulation of $>45^{\circ}$ ).

Design: This was a retrospective analytical study between January 2000 and December 2009.

Setting: The study was done at The Mombasa Hospital, Mombasa, Kenya.

Subjects: All skeletally mature patients with fractures of proximal humerus that were seen and managed by the author between January 2000 and December 2009 were included in the study. Three patients who had pathological fractures from tumour and infection were excluded from the study.

Results: Forty two out of 1454 fractures (2.9\%) were seen and managed for PHF during the study period. Twenty four were males and 18 females $(M: F$ ratio $=4: 3)$. Their ages ranged from 24 years to 85 years with a mean age of 54 years. However, the male mean age was 48 and 60 years for the females in the study. The commonest cause of injury was a fall $(71 \%)$ followed by motor vehicle accident (MVA) (17\%). Thirty one fractures $(74 \%)$ were a two-part fracture. Thirty two fractures $(74 \%)$ were treated by plate osteosynthesis out of which 25 (75\%) made satisfactory recovery. Five of thirty two operated cases (15\%) developed significant shoulder joint stiffness. Shoulder arthroplasty was unavailable.

Conclusion: Plate osteosynthesis is an effective method of treatment of selected patients with PHF. There is need for arthroplasty in those with severe injury, particularly those with osteoporosis.

\section{INTRODUCTION}

Proximal humerus fractures are relatively rare. Most of these fractures are reported to occur in elderly women over the age of 50 years, with the exception of isolated greater tuberosity fractures which occur in the younger individuals (1). In the elderly, the commonest cause is a fall while in the younger patients it is associated with vehicular accidents or sporting activity. Osteoporosis predisposes the elderly to this fragility fracture. Osteoporosis causes an increased risk of fracture through decreased bone mineral density, disruption of bone micro-architecture and reduction in noncollagenous proteins.

Proximal humerus fractures are difficult to manage, particularly in the osteoporotic bone, where the fracture is usually comminuted and the implants have poor purchase. Both operative and non operative methods are used in management of these injuries with different outcomes. Significant controversy continues regarding the best method of treating displaced fractures. In the past century, non-operative treatment of proximal humeral fractures was documented as an acceptable approach to treatment with modalities such as traction, manipulation and casting commonly employed (2,3). These surgeons emphasized on early functional range of motion and malunion was deemed acceptable (4). However, poor functional outcome especially in comminuted fractures was recognized by some authors (5).

In order to avoid this outcome, the tendency to open reduction and internal fixation became increasingly common. The race for osteosynthesis began in the late 1980's and by the last decade of the last century had become popular among traumatologists $(6,7)$. This change in approach came in tandem with new implant innovation, giving a quick succession to new implants, replacing the use of K-wires, staples and Rush pins with blade plates, locking plates and IM nails (8-10). IM fixation and plate/screws constructs have the greatest resistance to stress and torsional loading and are currently favoured $(11,12)$.

Metaphyseal fractures in osteoporotic bone are associated with specific fixation problems as the metaphyseal fragment is often very small. To improve fixation and resist bending forces a screw and plate construct with a locked angle between the plate and 
metaphyseal screw is often used. The locked plates with threaded screw holes in the plates create angular stability between the screws and the plates. However, shoulder surgeons recommend acute prosthetic replacement for fragmented fractures of proximal humerus particularly in the elderly (13).

Despite these efforts, various postoperative complications continue to be reported, the commonest being implant failure, nonunion, osteonecrosis of the humeral head and joint stiffness. Age has been identified as the most important prognostic factor for implant failure (14).

\section{MATERIALS AND METHODS}

This study was carried out at The Mombasa Hospital, a private hospital in Mombasa, Kenya. The clinical notes of all patients admitted and treated for PHF between the year 2000 and 2009 were retrieved for analysis. The relevant data was collected in a spreadsheets clinical research forms and analysed. The data collected included the patient's demography, cause of injury, mode of treatment, type of implant used and the immediate complications if any. Radiographs were reviewed again at the time of data collection in order to assess radiological osteoporosis and for fracture classification. These radiographs consisted of an AP and lateral views without special views or CT scans. In the postoperative follow up the study looked at the functional outcome and late complications.

Ineligible entrants included those patients with immature skeleton, pathological fractures and reoperations. The data was analysed using excel spreadsheets.

\section{RESULTS}

Between January 2000 and December 2009, a total of 1454 fractures were managed at The Mombasa Hospital. One hundred seventy eight (12\%) were humeral fractures (Figure 1). Forty five of the humeral fractures ( $3 \%$ of all fractures) were of the proximal humerus. Three patients were excluded from the study (two pathological fractures and one reoperation from a different set up).There were 24 males and 18 females (male to female ratio of 4:3).

The age distribution (Figure 2) shows a bimodal distribution with two peaks at about 50 years and 70 years. Male were aged between 18-71 years; 19 of them (79\%) were below 55 years of age (Table 1). On the contrary only four of the females (22\%) were below 55 years (Figure 3).

Table 2 shows the causes of injuries. Motor vehicle accidents, bicycle accidents and pedestrian injuries constituted $43 \%$ of the cases followed by a simple fall (36\%), which was the commonest cause in the elderly females. Fall from a height (including down the stairs, from riding camels in the beach and from construction sites) contributed $19 \%$ of the cases.

The injuries were classified according to the Neer classification. Table 3 shows the various fracture configurations according to this classification. The 2-part fracture pattern was the commonest (45\%).This involved a fracture through the surgical neck (SN) with a displacement of $>5 \mathrm{~mm}$ or/and ingulation of $>45^{\circ}$. A 3-part fracture through the surgical neck involving the greater tuberosity (GT) was the second commonest (29\%).Impacted fracture and those involving the lesser trochanter (LG) were rare.

Eight patients (24\%) were treated nonoperatively (Table 4). This involved using an armsling or body bandage. The remaining 32 patients had plate osteosynthesis and the commonest plate used was the clover-leaf plate (Synthes Inc. Switzerland) (75\%). Locking plates were used only in four patients.

Table 5 and Figure 4 show the outcome after osteosynthesis. Twenty four patients (75\%) recovered satisfactorily, five patients had joint stiffness (assessed as shoulder joint abduction and forward elevation of less than $90^{\circ}$ ) osteonecrosis of the humeral head and nonunion occurred in one patient each. One elderly lady died postoperative of renal failure.

Thenumberofpatients treated nonoperatively was considered small for analysis of outcome.

\section{DISCUSSION}

Fractures of the proximal humerus are relatively rare and represent no more than $3 \%$ of all upper extremity fractures and approximately $4 \%$ to $5 \%$ of all fractures. In this study they constituted $3 \%$ of all fractures seen.

Although they may occur in any age group, with the earliest noted at the time of birth, an increased frequency occurs in the elderly individuals because of the age-related increase in osteoporosis (15). The majorities of our patients was below 55 years and most were not fragility fractures.

Rose and colleagues showed that the incidence of proximal humeral fracture was lowest in the third decade of life and increased in both sexes until the age of 50 years. Thereafter, the incidence continued to increase, but the female-to-male ratio was 4:1 (16). They also showed that the greatest number of fractures in adult men appeared during the active ages between 30 and 60 years, whereas women a dramatic increase was noted after menopause (16).

Kristiansen and co-workers found that $77 \%$ of the fractures in all age groups occurred in women (17).

In this study there were more males than females. Most of the men were young, 79\% of them being below 55 years. Seventy eight percent of the females in this study were above 55 years of age. This illustrates the differences in the aetiology of injury between sexes and communities. 
In patients younger than 50 years, the most common causes of proximal humeral fractures are violent trauma such as falls from heights, motor vehicle accidents, and athletic injuries. High energy fractures from motor vehicle accidents and falling from heights were the commonest cause of injury in this study. This is in contrast to studies from developed countries where fragility fractures are the commonest. In fact, fractures from minimal to moderate trauma, such as a fall from the standing position or even direct impact were a distant second in contrast to reports elsewhere (18). Most of these low energy injuries were in the elderly females of Caucasian or Asiatic origin. Most of them fell while walking in the beach or in-doors. These injuries were rare among the Negroid population.

Proximal humeral fractures have been associated with various other injuries around the shoulder girdle. Acute rotator cuff tears can occur and have a significant impact on outcome. This injury is uncommon. In severe trauma, multiple fractures of the ipsilateral extremity may occur in addition to the proximal humeral fracture, posing challenges in management. Nerve injuries, particularly the infraclavicular brachial plexus and peripheral nerve branches have been reported.

Classification allows uniformity in reportage and research. Classification gives a guideline to the best treatment option. The four part Neer classification is the most widely used by OS today (19). Neer in 1975 modified and improved on Codman's observation of four major fracture fragments when proximal humerus fracture occurs. The classification takes into consideration the pattern and degree of displacement, other than the location of fracture lines (displacement of $>5 \mathrm{~mm}$ or angulation of $>45^{\circ}$ ). The main fragments are the articular head through the surgical neck (SN) or anatomical neck (AN), the greater tuberosity (GT), the lesser tuberosity (LT) and the humeral shaft. Neer's classic four part classification scheme is as follows:

1. 1-Part - Impacted fracture (through SN or AN)

2. 2-Part-angulated or displaced fracture separately involving the SN, AN, GT or LT

3. 3-Part- GT $+\mathrm{SN}$ or $\mathrm{LT}+\mathrm{SN}$

4. 4-Part-GT+SN+LT

Neer (19) also emphasized the prognostic importance of fracture dislocation, which has a higher Propensity to osteonecrosis and subsequent demand for arthroplasty.

Using this classification most of our fractures in this study had a Neer 2-part fracture. The next commonest was the 3-part fracture.Impacted fractures were there.

The aim of treatment is to restore the articular anatomy and its relationship to the tuberosities. The secondary aim is to address work and recreational demands in the younger individual.
The method of treatment will depend on fracture fragmentation, displacement bone quality and patients age. Minimally displaced fractures, impacted fractures and fractures in debilitated, elderly and those with severe osteopenia are managed non-operatively. A sling or body bandage is applied for two weeks. This is followed by pendulum shoulder exercises which gradually graduate to active shoulder motion in 4-6 weeks. Close follow up with weekly X-rays for the first 3 weeks is essential.

In this study only eight of our patients were treated in this manner, the rest were in the working age group, who sustained high energy fractures that required open reduction. The indications for surgical intervention are as follows:

The common two-part fractures are through the surgical neck or greater tuberosity. A displaced fracture of the greater tuberosity (usually associated with anterior dislocation), should be reduced and fixed to avoid malunion (20). Forty five percent of our patients had a two-part fracture mainly involving the surgical neck. They all had open reduction and internal fixation (Figure 5).

The commonest three-part fracture involves the SN and GT (Figure 6). When the quality of bone and the pattern of fracture allow open reduction and internal fixation with a proximal humeral locking plate is done. Thirty six percent of our patients were in this category and all had open reduction and internal fixation. However, elderly, osteopenic patients with fragmented fractures require primary arthroplasty where the facility is available (21).

In the typical four-part fracture the articular segment is usually dislocated anteriorly with the shaft sitting laterally and the tuberosities displaced. In a young patient, open reduction and fixation with a combination of plates, screws, heavy sutures etc is used while in the elderly, immediate hemiarthroplasty will be preferable (22). Only $14 \%$ of our patients were in this category, again they were treated with osteosynthesis. We had no ability for shoulder arthroplasty.

The associated injuries and long-term sequelae of fracture healing and joint injury can have a significant impact on outcome. Loss of humeral length with secondary deltoid weakening, traumatic arthritis, acute or chronic dislocations, rotator cuff tears with tuberosity displacement, nerve injuries, and vascular injuries add to the fracture complications.

The recorded complications after surgical management of proximal humeral fractures are nonunion, neurovascular damage, osteonecrosis, stiffness, arthritis and infection. This usually follows injury to the ascending branch of the anteriorcircumflex artery. Other complications are nonunion with implant failure and shoulder stiffness. The main complication of the operative approach is osteonecrosis (23). In this study the commonest complication was joint stiffness which constituted $16 \%$ of all operated patients. We 
suspect that we missed a few rotator cuff injuries that were not dressed during surgery. We had one case of osteonecrosis and one case of nonunion. One patient to had a pre-existing renal disease died of renal failure.

\section{CONCLUSION}

Fractures of the proximal humerus are uncommon but demanding in treatment. We have a different patient population from that described in the developed world. Whereas the majority of patients in those countries are elderly females, our patients were young and in their productive life. Motor vehicle accidents and occupational hazards are the common causes of injury in our population. The injury pattern is high energy requiring open reduction and internal fixation. Plate osteosynthesis is a reasonable approach with good results. Locking plates are a useful part of the orthopaedic armamentarium. There is also a small population of elderly people that will benefit from primary arthroplasty.

\section{REFERENCES}

1. Hulke, J.W. Injuries of the upper extremity. New York: William Wood, 1879.

2. Caldwell, J.A. and Smith, J. Treatment of unimpacted fractures of the surgical neck of the humerus. Am. J. Surg. 1936; 31: 141-144.

3. Frankau, C. A manipulative method for the reduction of fractures of the surgical neck of the humerus. Lancet. 1933; 2:755.

4. Mills, K.L. Severe injuries of the upper end of the humerus. Injury. 1974; 6:13-21.

5. Clifford, P.C. Fractures of the neck of the humerus: a review of the late results. Injury. 1980; 12: 91-95.

6. Weise, K., Meeder, P.J. and Wentzensen, A. Indications and operative technique in osteosyn thesis of fracture-dislocations of the shoulder joint in adults. Langenbecks Arch. Chir. 1980; 351: 91-98.

7. Zyto, K., Ahrengart, L., Sperber, A., et al. Treatment of displaced proximal humeral fractures in elderly patients. J. Bone Joint Surg. Br. 1997; 79: 412-417.

8. Stewart,M.J.and Hundley,J.M.Fractures of the humerus: a comparative study in methods of treatment. J. Bone Joint Surg. Am. 1955; 37: 681-692.
9. Habernek, H. and Orthner, E. A locking nail for fractures of the humerus. J. Bone Joint Surg. Br. 1991; 73: 651653.

10. Halder, S.C. and Chapman, J.A. Proximal humeral fractures: a new internal fixation device. J. Bone Joint Surg. Br. 1997; 79 [Suppl 4]: 408-409.

11. Clare,D.J.,Hersh,C.K.,Athanasiou,K.,etal.Biomechanical fixation strength in surgical neck fractures of the proximal humerus. Orthop Trans. 1998; 22:339.

12. Ruch, D.S. and Kuzma, G.R. Biomechanical rigidity following fixation of proximal humerus fractures. Orthop. Trans. 1998; 22: 599.

13. Hawkins, R.J. and Swjtlyk, P. Acute prosthetic replacement for severe fractures of the proximal mmerus. Clin. Orthop. 1993; 289:156-160.

14. Gerber, C., Werner, C.M. and Vienne, P. Internal fixation of complex fractures of the proximal humerus. Bone Joint Surg. Br. 2004; 86(6): 848-855.

15. Stimson, B.B. A Manual of Fractures and Dislocations, 2nd Ed. Philadelphia, Lea \& Febiger, 1947.

16. Rose,S.H.,Melton,L.J.,III.,Morrey,B.F.,etal.Epidemiologic features of humeral fractures. Clin. Orthop. 1982; 168: 24-30.

17. Kristiansen,B.,Barfod,G.,Bredesen,J.,etal.Epidemiology of proximal humeral fractures. Acta. Orthop. Scand. 1987; 58: 75-77.

18. Jensen, G.F., Christiansen, C., Boesen, J., et al. Relationship between bone mineral content and frequency of postmenopausal fractures. Acta. Med. Scand. 1983; 213: 61-63.

19. Neer, C.S. Four-segment classification of displaced proximal humeral fractures. AAOS Instr. Course Lect. 1975; 24: 160-168.

20. Marke.D.C.and Blasier, R.B.Bilateral anterior dislocation of the shoulders with greater tuberosity fractures. Orthopedics. 1994; 17: 945-949.

21. Gerber, A. and Warner, J.J.P. Hemiarthroplasty for management of complex proximal humerus fractures: preoperative planning and surgical solution. Philadelphia: Uppincott- Raven. 2005.

22. Wijgman, A.T., Roolker, W., Patt, I.W., et al. Open reduction and internal fixation of three- and fourpart fractures of the proximal part of the humerus. J. Bone Joint Surg. Am. 2002; 84(11): $1919-1925$.

23. Bellumore, Y., Determe, P., Bonnevialle, M., et al. Preliminary results of internal fixation combined with disto-proximal Kapandji nailing in fractures of the head and tuberosities of the humerus. J. Bone Joint Surg. Br. 1997;79: 45. 